\title{
Evolución de la prevalencia de caries y gingivitis en niños de 6 y 12 años de Peralillo, VI Región, entre el año 2000 y el 2010
}

\author{
Caries and gingivitis changes among 6 and 12 year-old children \\ of Peralillo, Chile, between 2000 and 2010
}

Cárdenas Espinoza $C^{1}$, Romero Saavedra $\mathrm{M}^{2}$, Giacaman Sarah RA ${ }^{3}$

\begin{abstract}
RESUMEN
Objetivo: Dada la limitada información epidemiológica en Chile sobre las patologías orales más prevalentes y el efecto de los programas de salud instaurados, el objetivo de este estudio fue evaluar los cambios en la prevalencia de caries y gingivitis en una población de 6 y 12 años de la VI Región, entre los años 2000 y 2010. Sujetos y Métodos: 143 sujetos de 6 y 12 años atendidos en el CESFAM de Peralillo fueron examinados clínicamente para detectar caries y diagnosticar gingivitis. Las fichas clínicas de 134 niños de las mismas edades y procedencia atendidos el año 2000 fueron analizadas para comparar los indicadores. Se obtuvo el índice de caries mediante el COPD y el ceod, además del diagnóstico gingival mediante parámetros clínicos. Los resultados de ambos años fueron comparados estadísticamente y las diferencias consideradas significativas si p <0.05. Resultados: La prevalencia de caries a los 6 años disminuyó de $89 \%$ a $65 \%$, pero sólo el COPD mostró una reducción estadísticamente significativa de 0.93 a 0.086 . A los 12 años, ni la prevalencia de caries ni el índice COPD mostraron un cambio significativo. La prevalencia de gingivitis disminuyó significativamente en ambos segmentos de edad en los 10 años de evaluación. Conclusiones: Pese a verificarse una leve disminución, la prevalencia de caries en Peralillo continua siendo muy alta y no ha variado sustancialmente en 10 años. Se sugieren más estudios similares para evaluar la eficacia de los programas en curso.
\end{abstract}

Rev. Clin. Periodoncia Implantol. Rehabil. Oral Vol. 4(3); 102-105, 2011.

Palabras clave: Índice COPD, ceod, prevalencia de caries, gingivitis, evolución de caries, riesgo de caries, factores de riesgo de caries.

\begin{abstract}
Aim: Given the limited epidemiological information in Chile on the most common oral diseases and the effectiveness of the existent programs, the aim of this study was to evaluate the changes in caries and gingivitis prevalence in a 6 and 12 year-old children from the VI Region, between the years 2000 and 2010. Subjects and Methods: 1436 and 12 year-old children treated at the CESFAM of Peralillo were clinically examined to detect and diagnose caries and gingivitis. Clinical records of 134 same ages children treated at the same service in the year 2000 were analyzed to compare the indexes. Caries index was obtained by DMFT and dmft and gingivitis was assessed by clinical parameters. Results obtained both years were compared and considered significant if $p<0.05$. Results: Caries prevalence decreased in the group of 6 year-olds from $89 \%$ to $65 \%$, but only DMFT showed a significant reduction from 0.93 to 0.086 . In the 12 year-old children, neither caries prevalence nor DMFT showed significant changes within the 10 -year period. Conclusions: In spite of a slight decrease, caries prevalence in Peralillo continues to be very high and it has not substantially changed in 10 years. Further similar studies are suggested to assess the efficacy of the current programs in use.
\end{abstract}

Rev. Clin. Periodoncia Implantol. Rehabil. Oral Vol. 4(3); 102-105, 2011.

Key words: DMFT, caries prevalence, gingivitis, caries evolution, caries changes, caries risk, caries risk factors.

\section{INTRODUCCIÓN}

La caries dental y la enfermedad gingival continúan siendo motivo de preocupación en Chile dada la alta tasa de prevalencia de estas enfermedades crónicas ${ }^{(1)}$. Pese a lo limitado de la información epidemiológica existente, se han reportado prevalencias de caries de $70.36 \%$ y de $62.5 \%$ en niños de 6 y 12 años, respectivamente ${ }^{(2)}$. La enfermedad gingival, por otra parte, también revela una muy alta prevalencia en la población, afectando al $55.09 \%$ y $66.9 \%$ de los niños de 6 y 12 años, respectivamente ${ }^{(2)}$.

La solución de la problemática de salud bucal en la población infantil requiere un enfoque multidisciplinario, basado en diagnósticos epidemiológicos apropiados. La crítica situación de salud bucal en muchos países del mundo ha llevado a la introducción del concepto de tratamiento con enfoque de riesgo para abordar eficientemente la situación ${ }^{(3)}$. La identificación de riesgo no sólo reside en factores biológicos, sino también debe considerar elementos psicosociales ${ }^{(4)}$. Dada la magnitud del problema y la escasez de recursos humanos y económicos para dar la adecuada cobertura, el año 2000 se definió priorizar la atención odontológica en los menores de 20 años y embarazadas primigestas, con el fin de lograr los objetivos sanitarios propuestos para la década $2000-2010^{(5)}$. Posteriormente se incluyó un tercer grupo de riesgo, los niños de 6 años para, de esta manera, incrementar el impacto de las medidas propuestas ${ }^{(6)}$. La decisión del priorizar los 6 años es ministerial y fundamentada en experiencias en otros países y en evidencia científica ${ }^{(7)}$. Las metas propuestas incluyen el logro de un 30\% de niños de 6 años son historia de caries para el año 2010 y un promedio de daño por caries (COPD) de 2.2 en adolescentes de 12 años de nivel socioeconómico bajo.

Peralillo es una comunidad predominantemente rural de la VI Región de Chile. Un estudio de prevalencia realizado con los niños beneficiarios del consultorio de Peralillo, el año 2000, mostró una muy alta prevalencia de caries y enfermedad gingival. Desde ese año, se han implementado medidas preventivas y terapéuticas tratando de dar cumplimiento a los programas sugeridos por el Ministerio de Salud. Dichos programas garantizan la atención odontológica integral de todos

1. Programa Odontológico. CESFAM Peralillo. VI Región, Chile.

2. Estadístico. CESFAM Peralillo. VI Región, Chile.

3. Unidad de Cariología. Departamento de Rehabilitación Buco-Máxilofacial. Universidad de Talca. Talca, Chile.

Correspondencia autor: Rodrigo A. Giacaman Sarah. giacaman@utalca.cl. Escuela de Odontología, Universidad de Talca. 2 Norte 685. Talca, Chile. Trabajo recibido el 01/06/2011. Aprobado para su publicación el 11/08/2011. Financiamiento: Esta investigación fue enteramente financiada con recursos institucionales del Centro de Salud Familiar de Peralillo, VI Región y del Departamento de Rehabilitación Buco-Máxilofacial de la Universidad de Talca y del Proyecto Fondecyt a RAG N¹1100005. 
los niños beneficiarios de 6 y 12 años ${ }^{(5)}$. Los resultados de dichas medidas, sin embargo, no han sido evaluados en el largo plazo. El presente estudio, por lo tanto, tiene por objeto comparar la prevalencia de caries y enfermedad gingival en los niños de 6 y 12 años de la comuna, luego de 10 años de implementados los programas de prevención y tratamiento.

\section{SUJETOS Y METODOLOGÍA}

La muestra en el estudio fue de 227 pacientes en total. Los sujetos analizados el año 2000 fueron 65 niños de 6 años de edad y 69 de 12 años. La muestra examinada el año 2010 consistió en 143 usuarios del CESFAM de Peralillo; 70 niños de 6 años y 73 de 12 años de edad. En ambos años, los niños provenían de Peralillo, Los Cardos y Población. Los participantes debían estar inscritos en el CESFAM y ser beneficiarios al año 2010 de los programas odontológicos de alta integral para 6 y 12 años. Se consideraron todos los niños de 6 años nacidos al 01 de enero de 2005 y los de 12 años nacidos al 01 de enero de 1999, de ambos sexos que cumplieran el criterio de ingreso. Fueron excluidos del estudio aquellos niños que presentaran alteraciones mentales y quienes presentaban dificultades mayores para la atención odontológica.

Se registró la información proveniente de las fichas clínicas de todos los pacientes atendidos el año 2000 en el CESFAM Peralillo, incluidas las postas de Población y Los Cardos. Las fichas incluían todos los datos necesarios para la comparación de las muestras con información detallada y fácilmente disponible en el registro del CESFAM. De cada una de las fichas se extrajeron los datos de las caries que presentaban en el primer examen del año 2000 , así como la presencia o ausencia de enfermedad gingival. Para el registro de los datos el año 2000 , el método de calibración consistió en elegir 10 sujetos de 6 años y 10 sujetos de 12 años, los que fueron examinados por dos odontólogos. Posteriormente, el índice kappa intraexaminador e interexaminador fue calculado y ambos fueron considerados calibrados si el valor alcanzaba al menos 0.9 . Los criterios diagnósticos para caries siguieron las recomendaciones internacionales ${ }^{(8)}$ al igual que los utilizados para determinar enfermedad gingival. Un niño fue considerado con gingivitis si presentaba al menos uno de los siguientes parámetros: 1) encía enrojecida, 2) encía aumentada de volumen asociada a biofilm oral visible ó 3) sangramiento al sondaje. Para la detección del biofilm se utilizó una pastilla reveladora de placa.

Los niños de 6 y 12 años del año 2010 fueron examinados clínicamente. El índice COPD y ceod de cada niño de 6 años y el COPD para los de 12 fueron obtenidos del examen practicado por tres odontólogos previamente calibrados ${ }^{(8)}$. El índice kappa fue de un 0.98 . El examen inicial fue realizado con un espejo dental sin aumento y sonda curva, bajo luz artificial de $5000^{\circ} \mathrm{K}$. El valor individual de cada índice correspondió a la suma de los dientes cariados, obturados y perdidos en ambas denticiones para los niños de 6 años y en la dentición permanente para los de 12. Para la determinación de la presencia o ausencia de gingivitis en los niños, se utilizaron parámetros clínicos de inflamación, similar a lo señalado para el año 2000. A pesar de ser obtenidos por examinadores distintos, en general los datos para el año 2000 y el 2010 fueron recolectados siguiendo los mismos criterios diagnósticos por odontólogos calibrados.

\section{Análisis Estadístico}

Los datos de caries fueron tabulados y procesados mediante SPSS (v. 15). Las diferencias en el COPD y ceod entre los datos del año 2000 y los del año 2010 fueron comparadas mediante el test $t$ de student. Las diferencias en el nivel de gingivitis fueron analizadas mediante el test chi cuadrado. Para ambas comparaciones, su utilizó un nivel de significancia del $95 \%$ y las diferencias fueron consideradas significativas si el valor "p" era menor que 0.05 .

\section{RESULTADOS}

\section{Distribución de la Muestra}

Un total de 277 niños fueron considerados en el estudio, incluidos los datos extraídos de las fichas del año 2000 y los examinados en el 2010. De los 70 niños participantes de 6 años en el 2010, un 59\% eran de Peralillo, $34 \%$ de Población y $7 \%$ de Los Cardos. El $61 \%$ de la muestra seleccionada correspondió al género masculino, y $39 \%$ correspondió al femenino (Tabla 1). Por su parte, 73 niños de 12 años participaron en el estudio el año 2010. 58\% de esos niños provenían de Peralillo, $30 \%$ de Población y $12 \%$ de Los Cardos. Un $49 \%$ de la muestra seleccionada eran de género masculino, mientras que el $51 \%$ correspondió al género femenino (Tabla 1). La distribución de la población de comparación del año 2000 se presenta igualmente en la Tabla 1.

Tabla 1. Número y distribución de los pacientes en la comuna de Peralillo según género y edad.

\begin{tabular}{|l|l|c|c|c|c|c|c|}
\hline \hline Edad & Año & Hombres & $\%$ & Mujeres & $\%$ & Total & $\%$ \\
\hline \multirow{2}{*}{6} & 2000 & 33 & 50.8 & 32 & 49.2 & 65 & 100 \\
\cline { 2 - 8 } & 2010 & 43 & 61.4 & 27 & 38.6 & 70 & 100 \\
\hline \multirow{2}{*}{12} & 2000 & 37 & 53.6 & 32 & 46.4 & 69 & 100 \\
\cline { 2 - 8 } & 2010 & 36 & 49.3 & 37 & 50.7 & 73 & 100 \\
\hline \multirow{2}{*}{} & Total & 149 & 53.8 & 128 & 46.2 & 277 & 100 \\
\hline
\end{tabular}

\section{Prevalencia de Caries}

En el año 2000, un $89.23 \%$ de los niños de 6 años de la comuna presentaban caries. El año 2010, este grupo etario disminuyó la prevalencia de caries a un $65.71 \%$ (Tabla 2). El grupo de 12 años, por su parte, no mostró ostensibles diferencias en la prevalencia de caries, con un $84.06 \%$ el año 2000 y $86.03 \%$ el 2010 (Tabla 2 ).

Tabla 2. Prevalencia de caries de los pacientes en la comuna de Peralillo en los años 2000 y 2010 , según edad.

\begin{tabular}{|l|l|c|c|c|c|c|c|}
\hline \hline Edad & Año & Sin Caries & $\%$ & Con Caries & $\%$ & Total & $\%$ \\
\hline \multirow{2}{*}{6} & 2000 & 7 & 10.77 & 58 & 89.23 & 65 & 100 \\
\cline { 2 - 8 } & 2010 & 24 & 34.29 & 46 & 65.71 & 70 & 100 \\
\hline \multirow{2}{*}{12} & 2000 & 11 & 15.94 & 58 & 84.06 & 69 & 100 \\
\cline { 2 - 8 } & 2010 & 10 & 13.70 & 63 & 86.30 & 73 & 100 \\
\hline \multirow{2}{*}{} & Total & 52 & 18.77 & 225 & 81.23 & 277 & 100 \\
\hline
\end{tabular}

Evolución de los Índices de Caries entre los Años 2000 y 2010

En los niños de 6 años, el ceod del año 2000 fue de 3.33 y el 2010 de 2.94. Pese a observarse una ligera disminución del índice, la diferencia no resultó estadísticamente significativa $(p<0.05)($ Figura $1 \mathrm{~A})$. Por el contrario, el COPD de este grupo de edad fue de $0.93( \pm 1.01)$ el año 2000 y de un $0.086( \pm 0.41)$ el año 2010 (Figura 1B). Al comparar ambos índices, se encontró una disminución significativa al cabo de 10 años $(p<0.05)$. En el caso de los niños de 12 años, se observó un COPD de $4.56( \pm 2.78)$ el 2000 y de $4.05( \pm 2.83)$ el 2010. La diferencia encontrada, sin embargo, no resultó estadísticamente significativa $(p>0.05)$ (Figura 2).

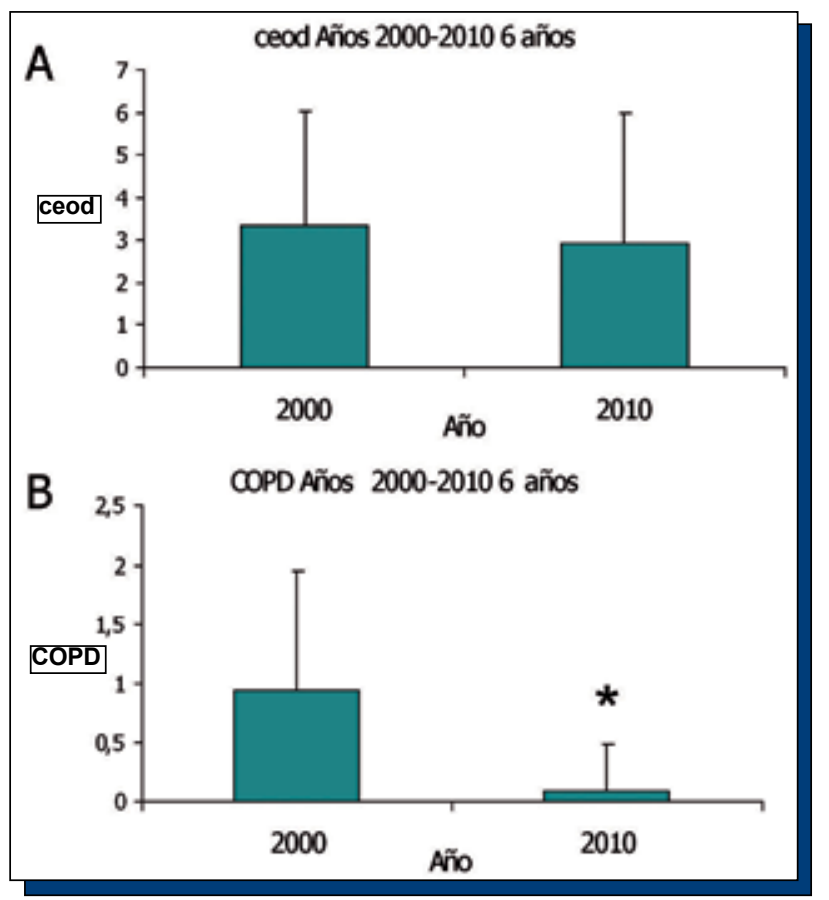

Figura 1. Índices de caries en niños de 6 años. La figura muestra los índices ceod (A) y COPD (B) del grupo de niños de 6 años de Peralillo, según el año de evaluación. Las barras representan el promedio del índice correspondiente \pm DS. *: $p<0.05$. 


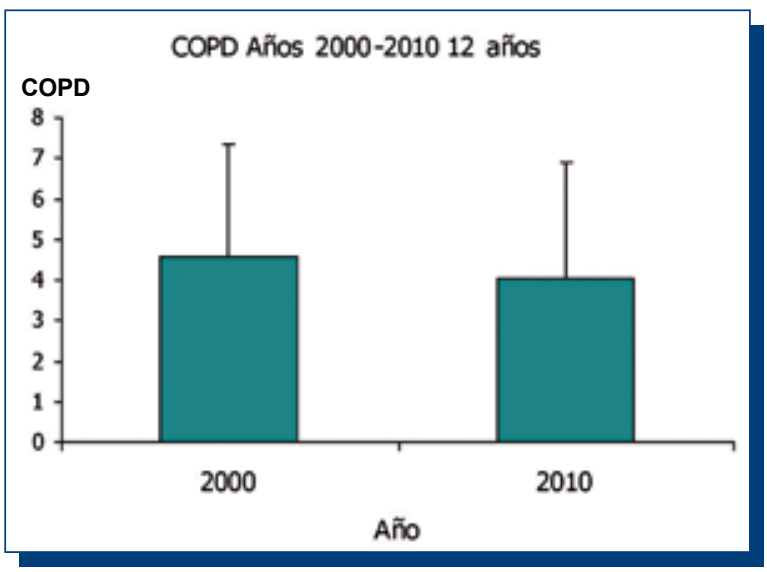

Figura 2. Índice de caries en niños de 12 años. La figura muestra el índice COPD de los niños de 12 años de Peralillo, según el año de evaluación. Las barras representan el promedio del COPD \pm DS.

\section{Prevalencia de Gingivitis}

En los niños de 6 años se detectó la presencia de gingivitis en un $90.77 \%$ el año 2000 (Figura 3A). La prevalencia en este grupo etario disminuyó a $57.14 \%$ el año $2010(p<0.05)$ (Figura 3A). En el caso de los niños de 12 años, la prevalencia de gingivitis alcanzó a un $82.61 \%$ el año 2000 y disminuyó significativamente a un $76.71 \%$ para el $2010(p<0.05)$ (Figura 3B).

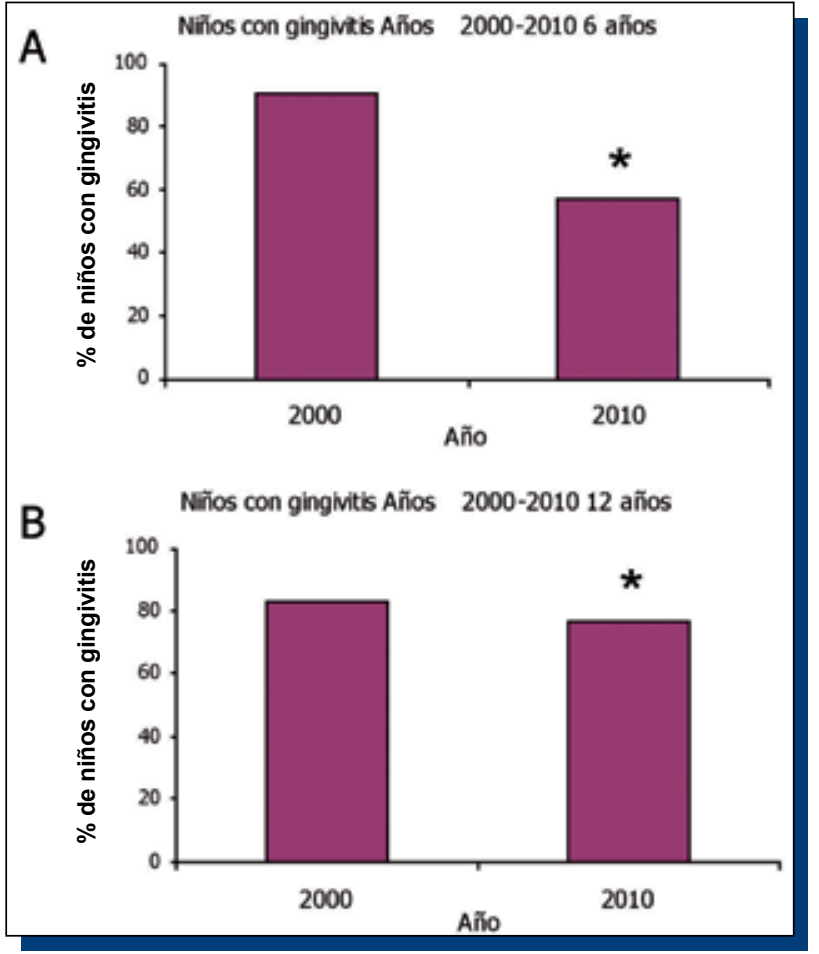

Figura 3. Prevalencia de gingivitis en niños de 6 y 12 años. La figura grafica la prevalencia de gingivitis en los niños de 6 años (A) y de 12 años (B) de la comuna de Peralillo, según el año de evaluación. Las barras representan la prevalencia de gingivitis al momento del examen. *: $p<0.05$.

\section{DISCUSIÓN}

A pesar de observarse una leve disminución en los índices de caries y enfermedad gingival, la prevalencia de estas patologías continúa siendo muy alta en la comuna de Peralillo. Pese a lo alto de la prevalencia de caries, se ha experimentado una considerable baja en ella desde el 2000 al 2010; pasando de más de $89 \%$ a cerca de $65 \%$ en los niños de 6 años, lo que incluso es inferior a la prevalencia nacional reportada para esa edad de cerca del $70 \%{ }^{(2)}$. La situación a los 12 años, sin embargo, no ha variado demasiado desde el 2000 al 2010. La prevalencia de caries en la población de Peralillo se ha mantenido constante en este grupo etario. En cuanto a los índices de caries en los 6 años, el ceod no varió significativamente en la comuna en 10 años, a pesar que los valores del índice en ambos años son inferiores al valor reportado para Chile en $2007^{(2)}$. Por otra parte, resulta promisorio que el COPD a los 6 años muestre una clara disminución a los 10 años, situándose además muy por debajo del promedio nacional reportado(2). Para los 12 años de edad, el índice COPD no sufrió variaciones significativas en 10 años, ubicándose alrededor de 4 . A esa edad, resulta muy interesante notar que el COPD es más del doble más alto que el del país(5).

En el caso de la gingivitis, los niños experimentaron una disminución en la prevalencia desde el 2000 al 2010. La reducción de la prevalencia fue más dramática en los niños de 6 años. Se ha reportado una tendencia mundial a una reducción de caries $^{(9)}$. Dicha reducción, sin embargo, se asocia directamente con el ingreso per cápita del país ${ }^{(10)}$. En ese sentido, Chile es aún considerado un país en desarrollo y enfrenta, por ende, el desafío de reducir su nivel de caries actual. Peralillo es una comuna de la VI Región con una población rural menor a la urbana, pero mayor que la media del país ${ }^{(11)}$. Adicionalmente, la comuna de Peralillo posee indicadores de pobreza, analfabetismo y años de escolaridad por debajo de la media de Chile ${ }^{(11)}$. Recientes investigaciones han alertado sobre el rol que los factores sociales juegan en la caries dental. De hecho, en un reciente estudio de determinación de riesgo en niños, los factores sociales de ingreso y educación resultaron tan estrechamente relacionados con caries como algunos factores biológicos ${ }^{(3)}$. El hecho que los niños de Peralillo no hayan experimentado un cambio más decidido en los indicadores de caries en 10 años, puede deberse precisamente a la situación más acentuada de pobreza y menores indicadores de educación.

La comuna de Peralillo podría representar a varias otras del Chile central, con un grado de pobreza mayor que la media del país y una alta proporción de población rural. Un bajo ingreso económico y factores sociales explican un bajo acceso a los servicios de salud y han sido asociados con mayores valores predictivos de caries, progresión de lesiones y mayor incidencia de caries en infantes y niños ${ }^{(4,12,13)}$. En efecto, Peralillo presenta una muy alta carga de enfermedad bucal. Pese a lo alto de las tasas de caries y gingivitis observadas en la comuna en los años de observación, se reconocen algunas mejoras en los índices utilizados, con un énfasis en los 6 años en la dentición permanente. Las acciones que se realizan en el modelo de atención integral utilizado desde el año 2000 en adelante en la comuna de Peralillo incluyen; alta integral a los grupos priorizados por el MINSAL: niños de 6 y 12 años, embarazadas y menores de 20 años. A los 10 cupos semanales para los programas priorizados de 6 y 12 años se les confecciona una ficha clínica y se les realiza educación en técnicas de higiene y consejo dietético. En la siguiente sesión se efectúa profilaxis y en las sucesivas se realizan sellantes de primeros y segundos molares y restauraciones acorde a las necesidades. En los grupos de bajo riesgo cariogénico se realizan terapias de flúor barniz al $5 \%$ en dos sesiones cada 6 meses por 1 año. Asimismo, se realizan actividades educativas grupales dos veces al año, abordando temáticas sobre etiología de caries y autocuidado.

En los últimos años en Chile, el Ministerio de Salud ha hecho un esfuerzo en implementar y consolidar programas de prevención en la comunidad(5). Es, entonces, razonable concebir que la mejora en la prevalencia de caries y la disminución en la gingivitis se deba a los resultados positivos de estos esfuerzos. Adicionalmente, el uso de fluoruros ha mostrado ser una eficaz manera de prevenir la caries en el mundo entero, independientemente del nivel socioeconómico u otras variables implicadas en el proceso(14). El aumento en el acceso a agua potable fluorada, un mayor nivel de ingresos y el aumento en la cobertura de atención odontológica en la comuna, puede explicar la disminución observada.

Pese a lo auspicioso de la disminución observada en los niños de 6 años en los 10 años de observación, el hecho que los niños de 12 años no varíen sustancialmente sus índices permanece como una preocupación. Razones para este estancamiento a los 12 años puede deberse a que los esfuerzos preventivos y curativos se concentran a los 6 años y luego se retoman a los 12 años, tiempo talvez muy extenso para mantener cambios conductuales sostenibles, particularmente en lugares que además enfrentan desmedro en sus condiciones socioculturales y en donde la familia puede no reforzar los cambios esperados. Factores deletéreos de dieta y conductuales desde los 6 a los 12 años, pueden enmascarar los beneficios otorgados por los programas implementados a los 6 años. Experiencias en Europa Occidental han mostrado que 
una mejora en los hábitos de cepillado en la población han llevado a una disminución del COPD desde 3.0 a $1.0^{(9)}$. Dicha situación puede además explicar la baja en la prevalencia de gingivitis en la población de este estudio a los 12 años. Además de continuar con los programas existentes, se debería considerar incrementar el acceso a salud bucal de niños en las edades intermedias de 8 y 10 años, para así evitar la pérdida de continuidad en los programas implementados.

Por último, las variaciones en los indicadores epidemiológicos de caries sugieren un monitoreo permanente, subrayando la importancia de los estudios epidemiológicos en salud bucal. En la experiencia de otros países, incluyendo los modelos más exitosos, pese a disminuciones de caries en el tiempo, llega un momento en que la tendencia a la disminución se detiene ${ }^{(9)}$. Factores poblacionales como inmigración o cambios en los estilos de vida, pueden incrementar, o al menos estancar la disminución en la prevalencia de caries o enfermedad gingival, en poblaciones con bajas tasas ${ }^{(9)}$. Basándose en los resultados obtenidos en este estudio y en los alarmantes índices de enfermedades bucales, se sugieren nuevos estudios similares para dimensionar el impacto que han tenido las políticas de salud bucal en Chile.

\section{CONFLICTOS DE INTERÉS}

Los autores declaran no tener ninguna vinculación financiera en relación con cualquiera de los productos involucrados en este estudio.

\section{REFERENCIAS BIBLIOGRÁFICAS}

1. MINSAL. Encuesta Nacional de Salud (ENS). 2003, Chile.

2. MINSAL. Soto $L$, Tapia $R$ et al. Diagnóstico nacional de salud bucal del niño de 6 años. MINSAL, 2007. Chile.

3. Fontana M, Jackson R, Eckert G, Swigonski N, Chin J, Zandona AF, Ando M, Stookey GK, Downs S, Zero DT. Identification of caries risk factors in toddlers. J Dent Res, 2011; 90(2): 209-214.

4. Eckert GJ, Jackson R, Fontana M. Sociodemographic variation of caries risk factors in toddlers and caregivers. Int J Dent, 2010.

5. Soto L, Tapia R, Jara G, Rodríguez G, Urbina T. Diagnóstico Nacional de Salud Bucal del Adolescente de 12 años y Evaluación del Grado de Cumplimiento de los Objetivos Sanitarios de Salud Bucal 2000-2010. Universidad Mayor. Santiago, Chile. 2007.

6. Badenier O, Moya R, Cueto A. Prevalencia de las enfermedades buco dentales y necesidades de tratamiento en la V Región. Proyecto FONIS. 2007. 7. MINSAL. Guía Clínica Salud Oral Integral en Niños de 6 años. $1^{\text {era }}$ Ed. Santiago, MINSAL. 2005.

8. WHO. World Health Organization. Oral health surveys: Basic methods Geneva. $4^{\text {th }}$ ed. 1997
9. Marthaler TM. Changes in dental caries 1953-2003. Caries Res, 2004; 38(3): 173-181.

10. Bernabe $\mathrm{E}, \mathrm{Hobdell} \mathrm{MH}$. Is income inequality related to childhood dental caries in rich countries? J Am Dent Assoc, 2010; 141(2): 143-149.

11. Biblioteca del Congreso. Reporte Estadístico Regional, Peralillo. Sistema Integrado de Información Territorial. 2008. Accedido 20 de abril de 2011. Disponible en: http://siit.bcn.cl

12. Hallett KB, O'Rourke PK. Social and behavioural determinants of early childhood caries. Aust Dent J, 2003; 48(1): 27-33.

13. Martens L, Vanobbergen J, Willems S, Aps J, De Maeseneer J. Determinants of early childhood caries in a group of inner-city children. Quintessence Int, 2006; 37(7): 527-536.

14. Marinho VC. Cochrane reviews of randomized trials of fluoride therapies for preventing dental caries. Eur Arch Paediatr Dent, 2009; 10(3): 183-191. 